\title{
Free Vibration Characteristics of a Thin-Walled Ring Under Different Boundary Conditions
}

\author{
Abedinilaksar M. Javad, Yang Jianming* \\ Faculty of Engineering and Applied Science, Memorial University, \\ St. John's, NL, Canada A1B-3X5 \\ *jyang@mun.ca
}

\begin{abstract}
This paper investigates the natural frequencies of a thin-walled Euler-Bernoulli ring under different boundary conditions with an analytical method. The free in-plane vibration problem of the system is solved in this work and analytical results are validated with numerical solutions obtained from simulations done in ABAQUS package. Comparing the cases of hinged and fixed supports, both analytical and FEM methods confirmed that the natural frequencies for the case of having fixed supports are considerably higher.
\end{abstract}

Keywords: Free Vibration, Mode Shape, Natural Frequency, Euler-Bernoulli Beam, Ring Vibration.

\section{INTRODUCTION}

Dynamic characteristics of free rings have been investigated in many works [1-6], and closed-form equations for evaluating natural frequencies and associated mode shapes are already obtained. However, there are few authors investigating the dynamic characteristics of rings on radial supports [7-9]. The vibration of a thin ring has been studied extensively by Rao [2]. Wu and Parker [3] used perturbation and Galerkin methods to study the mode contamination of a ring with an elastic foundation. Zhang et al. [4] studied the natural frequencies and mode contaminations of a spinning ring. Rao et al. [10] determined natural frequencies and associated mode shapes of a ring on rigid radial support using the differential equation formulation. However, their numerical results seem to be only accurate for a small range of radial supports. For cases with small or large numbers of radial supports their method was not entirely satisfactory. Sahay et al. [11] studied the same problem using a different method and presented a solution for the case of having a large number of radial supports. Transfer matrix is another approach that has been used by a few other researchers [12-14] to express more accurate results for natural frequencies and modes for a ring supported by numerous radial supports. However, some mode shapes neglected because they assumed the total vibrational mode of the whole ring could be determined by considering the same behavior of one studied segment for all the other segments. This behavior is only feasible for a nongeneral case of boundary conditions. Malik et al. [15] used the wave approach and determined the natural frequencies from the propagation constants of the ring, assuming it as an endless periodic structure. Zakrzhevskii et al. [16] used a numerical method to solve a boundary-value problem (BVP) for an elastic ring fixed at a point. However, none of those works could express a neat closed-form formulation for mode shapes of a ring with different types of radial supports. To fill this knowledge gap, this paper investigates the free vibration of a ring with various different supports, including hinged and fixed supports. Studying dynamics and free vibration of thin-walled rings play an important role in the functional performance of many systems that undergo complex behavior when subjected to static or moving loads. For instance, studying the vibrations of rings used in the planetary gear train is a critical consideration, particularly for high speed and heavy load applications. Planetary gear trains are widely used in many applications, such as automobiles, helicopters, and wind turbines, etc. This wide range of applications shows the importance of analyzing the vibration of a typical ring under different possible boundary conditions.

The paper is organized below. In section II, the in-plane vibrational equations are derived. Mode shapes are boundary conditions corresponding to different support are discussed in section III and IV. Conclusions are given in section V, and some recommendations for future work are outlined in section VI.

\section{DYNAMIC MODEL}

Figure 1 shows a thin-walled ring represented by its centerline. To avoid the complexity in the governing equations, the rigidity of the ring $E I$ is assumed constant, and the effects of rotary inertia and shear deformation are both neglected. The ring is being held with $n$ radial supports as shown in the figure

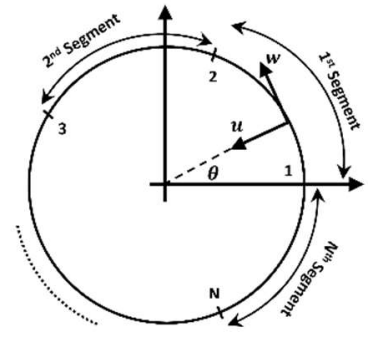

Figure 1. A thin-walled ring and the location of each support 


\section{A. System Equations}

To derive the governing equations, a typical element at an arbitrary position $\theta$ is selected. The forces and moments acting on this element can be expressed as functions of deformation components. Neglecting the small quantities of high order terms will result in the following equilibrium equations in tangential $w$, radial $u$, and rotational motion $\varphi$ around the $w$ axis.

$$
\begin{gathered}
\frac{\partial Q}{\partial \theta}+N=\rho A R \ddot{u} \\
\frac{\partial N}{\partial \theta}-Q=\rho A R \ddot{W} \\
\frac{\partial M}{\partial \theta}+R \cdot Q=\rho I R \ddot{\varphi}
\end{gathered}
$$

where $\mathrm{R}$ is the radius of ring centerline, $\rho$ is the total mass density, $\mathrm{A}$ is the cross-sectional area, I is the moment of inertia of the area $A$ about w axis. The normal force $\mathrm{N}$ is considered positive in tension, the shear force $Q$ is considered positive when acting in a radially inward direction on a positive face, and finally, the in-plane bending moment $\mathrm{M}$ is considered positive when it tends to reduce the curvature of the ring. Similarly, the relationship between the displacement functions can be represented as follows:

$$
\begin{gathered}
\varphi=\frac{1}{R}\left(\frac{\partial u}{\partial \theta}+w\right) \\
N=\frac{E A}{R}\left(\frac{\partial w}{\partial \theta}-u\right)-\frac{M}{R} \\
M=\frac{E I}{R}\left(\frac{\partial \varphi}{\partial \theta}\right)
\end{gathered}
$$

Equation (6) can alternatively be presented based on the tangential and radial displacements by applying equation 4 into that. This new relationship is shown in (7).

$$
M=\frac{E I}{R^{2}}\left(\frac{\partial^{2} u}{\partial \theta^{2}}+\frac{\partial w}{\partial \theta}\right)
$$

Using Hooke's law, the axial stress $\sigma_{0}$ can be related to the strain $\varepsilon$ which can be alternatively written based on the radial and tangential displacements [2].

$$
\varepsilon=\frac{1}{R}\left(\frac{\partial w}{\partial \theta}-u+\frac{x}{R} \frac{\partial}{\partial \theta}\left(w+\frac{\partial u}{\partial \theta}\right)\right)
$$

where $\mathrm{x}$ is the distance from the ring centerline.

This equation can be simplified for the centerline since it has assumed to remain unstretched. Hence, by considering $\varepsilon=0$ for the centerline and neglecting the effects of products of tiny quantities in (8), the tangential and radial displacements can be related as follows:

$$
u=\frac{\partial w}{\partial \theta}
$$

The equation (9) is called the inextensibility condition. Under this condition, the right-hand side of (3) would be zero due to zero deflection for the ring centerline in the tangential direction. In order to find a relationship for the shear force $\mathrm{Q}$, first, the normal force $\mathrm{N}$ needs to be found from (1) and replaced into (2).
Then, by substituting (3) and (9) into the obtained equation, the following relationship will be derived.

$$
Q=-\rho A R \ddot{w}-\frac{1}{R} \frac{\partial M}{\partial \theta}
$$

Eventually, by combining (1) through to (5), (7), (9), and (10) into a single equation, the in-plane flexural classical vibration equation for a thin ring can be represented as follows:

$$
\begin{gathered}
\frac{\partial^{6} w(\theta, t)}{\partial \theta^{6}}+2 \frac{\partial^{4} w(\theta, t)}{\partial \theta^{4}}+\frac{\partial^{2} w(\theta, t)}{\partial \theta^{2}}+\frac{R^{4} \rho A}{E I} \frac{\partial^{2}}{\partial t^{2}}\left(\frac{\partial^{2} w(\theta, t)}{\partial \theta^{2}}-\right. \\
w(\theta, t))=0
\end{gathered}
$$

where $w(\theta, t)$ is the tangential displacement of the centerline of the ring. By separating the variables using the form represented in (12), the total solution can be written as multiplication of mode shape function $\Psi(\theta)$ by time-varying harmonic function $\mathrm{q}(\mathrm{t})$ as follows:

$$
\begin{aligned}
w(\theta, t)= & \sum_{j=1}^{\infty} C_{j} \Psi_{j}(\theta) \mathrm{q}_{j}(t) \simeq \Psi(\theta) q(t) \\
& \mathrm{q}_{j}(t)=B_{j} \exp \left(i \omega_{j} t\right)
\end{aligned}
$$

Similarly, for the other two displacement functions, in general, the following equations are valid.

$$
\begin{aligned}
& u(\theta, t)=\sum_{j=1}^{\infty} D_{j} U_{j}(\theta) \mathrm{q}_{j}(t) \simeq U(\theta) q(t) \\
& \varphi(\theta, t)=\sum_{j=1}^{\infty} E_{j} \Phi_{j}(\theta) \mathrm{q}_{j}(t) \simeq \Phi(\theta) q(t)
\end{aligned}
$$

where $\mathrm{i}$ is the unit imaginary number $i=\sqrt{-1}$. For simplicity in future substitution, just the first order $(j=1)$ is considered throughout this paper. The equation (12) can now be plugged into the governing vibrational, i.e. (11). This substitution will result in:

$$
\begin{gathered}
\frac{\partial^{6}}{\partial \theta^{6}} \Psi(\theta) q(t)+2 \frac{\partial^{4}}{\partial \theta^{4}} \Psi(\theta) q(t)+\frac{\partial^{2}}{\partial \theta^{2}} \Psi(\theta) q(t)+ \\
\frac{R^{4} \rho A}{E I}\left(\frac{\partial^{2} \Psi(\theta)}{\partial \theta^{2}}-\Psi(\theta)\right) \ddot{q}(t)=0
\end{gathered}
$$

By substituting (13) into the (16), (17) will be derived, as follows:

$$
\begin{gathered}
\left(\frac{\partial^{6} \Psi(\theta)}{\partial \theta^{6}}+2 \frac{\partial^{4} \Psi(\theta)}{\partial \theta^{4}}+\frac{\partial^{2} \Psi(\theta)}{\partial \theta^{2}}\right) \exp (i \omega t)-\frac{R^{4} \rho A \omega^{2}}{E I}\left(\frac{\partial^{2} \Psi(\theta)}{\partial \theta^{2}}-\right. \\
\Psi(\theta)) \exp (i \omega t)=0
\end{gathered}
$$

Equation (18) is a neat form of the (17) after eliminating the time-variable exponential part from all terms of it.

$$
\frac{\partial^{6} \Psi(\theta)}{\partial \theta^{6}}+a \frac{\partial^{4} \Psi(\theta)}{\partial \theta^{4}}+b \frac{\partial^{2} \Psi(\theta)}{\partial \theta^{2}}+c=0
$$

where:

$$
a=2
$$




$$
\begin{gathered}
b=1-\Omega^{2} \\
c=\Omega^{2} \\
\Omega=R^{2} \sqrt{\frac{\rho A}{E I}} \omega
\end{gathered}
$$

From (9), the relationship between radial and tangential mode shapes is as follows:

$$
U=\frac{\partial \Psi}{\partial \theta}
$$

Similarly, by means of (4) and (9), the relationship between mode shapes of rotational and tangential displacement functions can be expressed as follows:

$$
\Phi=\frac{1}{R}\left(\frac{\partial^{2} \Psi}{\partial \theta^{2}}+\Psi\right)
$$

\section{MODE SHAPE DETERMINATION}

The general solution of (18) takes the form of:

$$
\Psi(\theta)=Z e^{\Upsilon \theta}
$$

Inserting (25) and its derivatives into (18) forms a sixth-order algebraic equation as below:

$$
\Upsilon^{6}+a \Upsilon^{4}+b \Upsilon^{2}+c=0
$$

This equation can be simplified into a cubic equation in (27):

$$
\lambda^{3}+a \lambda^{2}+b \lambda+c=0
$$

where $\lambda=\Upsilon^{2}$. Thus, the six roots of (26) can be found after the determination of the three roots of (27) i.e. $\lambda_{r}(r=1,2,3)$. The advantage of this method over solving the original sixth order equation is the roots can make closed-form shapes. Once $\lambda_{r}$ are calculated, $\Upsilon_{s}(s=1-6)$ can be easily obtained through $\Upsilon_{s}=$ $\pm \sqrt{\lambda_{r}}$.

In order to solve any standard cubic equation, a discriminant parameter $\Delta$ needs to be defined. This parameter is expressed in (27) [17].

$$
\Delta=\bar{Q}^{3}+\bar{R}^{2}
$$

where:

$$
\begin{gathered}
\overline{\mathrm{Q}}=\frac{1}{9}\left(3 b-a^{2}\right) \\
\overline{\mathrm{R}}=\frac{1}{54}\left(9 a b-27 c-2 a^{3}\right)
\end{gathered}
$$

Having defined $\Delta$, the roots of (27) can be expressed as follows:

$$
\begin{gathered}
\lambda_{1}=\mathrm{S}+\mathrm{T}-\frac{a}{3} \\
\lambda_{2}=-\frac{1}{2}(\mathrm{~S}+\mathrm{T})-\frac{a}{3}+i\left(\frac{\sqrt{3}}{2}(\mathrm{~S}-\mathrm{T})\right) \\
\lambda_{3}=-\frac{1}{2}(\mathrm{~S}+\mathrm{T})+\frac{a}{3}+i\left(\frac{\sqrt{3}}{2}(\mathrm{~S}-\mathrm{T})\right)
\end{gathered}
$$

where:

$$
\begin{aligned}
& \mathrm{S}=\sqrt[3]{\bar{R}+\sqrt{\Delta}} \\
& \mathrm{T}=\sqrt[3]{\bar{R}-\sqrt{\Delta}}
\end{aligned}
$$

Thus, (25) can be rewritten based on the roots evaluated from (30) through (32), as follows:

$$
\begin{gathered}
\Psi(\theta)=\sum_{i=1}^{6} A_{i} e^{\Upsilon_{i} \theta}=A_{1} e^{\Upsilon_{1} \theta}+\mathrm{A}_{2} \mathrm{e}^{\Upsilon_{2} \theta}+\mathrm{A}_{3} \mathrm{e}^{\Upsilon_{3} \theta}+ \\
\mathrm{A}_{4} \mathrm{e}^{\Upsilon_{4} \theta}+\mathrm{A}_{5} \mathrm{e}^{\Upsilon_{5} \theta}+\mathrm{A}_{6} \mathrm{e}^{\Upsilon_{6} \theta}
\end{gathered}
$$

Or alternatively:

$$
\begin{aligned}
\Psi(\theta)=A_{1} e^{\sqrt{\lambda_{1}} \theta}+ & A_{2} e^{-\sqrt{\lambda_{1}} \theta}+A_{3} e^{\sqrt{\lambda_{2}} \theta}+A_{4} e^{-\sqrt{\lambda_{2}} \theta} \\
& +A_{5} e^{\sqrt{\lambda_{3}} \theta}+A_{6} e^{-\sqrt{\lambda_{3}} \theta}
\end{aligned}
$$

The roots of (27) i.e. $\lambda_{r}(r=1,2,3)$ can take different forms based on the discriminant $\Delta$. For $\Delta>0$, there will be one real root and the other two roots will be complex conjugates. For the case of $\Delta=0$, all three roots will be real; however, two of them will be identical. In other words, there will be a set of repeated roots in the system. In this case, the corresponding terms of (36) associated with the repeated roots will take forms of $\left(A_{\text {rep }}+\right.$ $\left.A_{\text {rep }+1} \theta\right) \exp \left(\sqrt{\lambda_{\text {rep }}} \theta\right)$. Finally, for $\Delta<0$, all roots will be real and distinct. For this system, the discriminant is expressed below:

$$
\Delta=-\frac{c^{3}}{27}+\frac{71 c^{2}}{108}-\frac{2 c}{27}
$$

\section{BOUNDARY CONDITIONS}

For the ring studied in the current work, three common supports are considered. Firstly, it is assumed that the ring is hinged to its frame. Secondly, $n$ fixed supports are considered to hold the ring along its rim. Finally, the ring is considered to be connected to its frame at $n$ equally-spaced points. By assuming equal length for all segments, the angle between any two connected segments $\zeta$ can be expressed as follows

$$
\zeta=\frac{2 \pi}{n} \quad(\mathrm{rad})
$$

\section{A. Hinged Supports}

Based on the radial-tangential $\mathrm{u}-\mathrm{w}$ coordinate demonstrated in Fig. 1, the slope and bending moment of the ring can be represented as $\frac{\partial^{2} w}{\partial \theta^{2}}$ and $\frac{\partial^{3} w}{\partial \theta^{3}}$, respectively. At each support, normal and tangential deflections are zero $u=w=0$. In this scenario, the moment is zero at each node. For an arbitrary support i one can say:

$$
\begin{gathered}
u_{i}\left(\theta_{i}\right)=0 \\
w_{i}\left(\theta_{i}\right)=0 \\
\left.\frac{\partial^{2} w}{\partial \theta^{2}}\right]_{\theta=\theta_{i}}=0
\end{gathered}
$$

Applying (39) through (41) into (36) for the first segment leads to the following equations. This can be similarly re-written for the first scenario. 
Zero tangential deflection at each support:

$$
\begin{gathered}
\Psi(0)=A_{1}+A_{2}+A_{3}+A_{4}+A_{5}+A_{6}=0 \\
\Psi(\zeta)=A_{1} e^{\sqrt{\lambda_{1}} \zeta}+A_{2} e^{-\sqrt{\lambda_{1}} \zeta}+A_{3} e^{\sqrt{\lambda_{2}} \zeta}+A_{4} e^{-\sqrt{\lambda_{2}} \zeta}+ \\
A_{5} e^{\sqrt{\lambda_{3}} \zeta}+A_{6} e^{-\sqrt{\lambda_{3}} \zeta}=0
\end{gathered}
$$

Zero radial deflection at each support:

$$
\begin{gathered}
\left.\frac{d \Psi(\theta)}{d \theta}\right]_{\theta=0}=\sqrt{\lambda_{1}} A_{1}-\sqrt{\lambda_{1}} A_{2}+\sqrt{\lambda_{2}} A_{3}-\sqrt{\lambda_{2}} A_{4}+\sqrt{\lambda_{3}} A_{5}- \\
\sqrt{\lambda_{3}} A_{6}=0 \\
\left.\frac{d \Psi(\theta)}{d \theta}\right]_{\theta=\zeta}=\sqrt{\lambda_{1}} A_{1} e^{\sqrt{\lambda_{1}} \zeta}-\sqrt{\lambda_{1}} A_{2} e^{-\sqrt{\lambda_{1}} \zeta}+\sqrt{\lambda_{2}} A_{3} e^{\sqrt{\lambda_{2}} \zeta}- \\
\sqrt{\lambda_{2}} A_{4} e^{-\sqrt{\lambda_{2}} \zeta}+\sqrt{\lambda_{3}} A_{5} e^{\sqrt{\lambda_{3}} \zeta}-\sqrt{\lambda_{3}} A_{6} e^{-\sqrt{\lambda_{3}} \zeta}=0
\end{gathered}
$$

Zero moment at each support:

$$
\begin{gathered}
\left.\frac{d^{3} \Psi(\theta)}{d \theta^{3}}\right]_{\theta=0}=\sqrt{\lambda_{1}{ }^{3} A_{1}}-\sqrt{\lambda_{1}{ }^{3} A_{2}}+\sqrt{\lambda_{2}{ }^{3}} A_{3}-\sqrt{\lambda_{2}{ }^{3}} A_{4}+ \\
\sqrt{\lambda_{3}{ }^{3} A_{5}-\sqrt{\lambda_{3}{ }^{3}} A_{6}=0} \\
\left.\frac{d^{3} \Psi(\theta)}{d \theta^{3}}\right]_{\theta=\zeta}=\sqrt{\lambda_{1}{ }^{3}} A_{1} e^{\sqrt{\lambda_{1}} \zeta}-\sqrt{\lambda_{1}{ }^{3}} A_{2} e^{-\sqrt{\lambda_{1}} \zeta}+ \\
\sqrt{\lambda_{2}{ }^{3} A_{3}} e^{\sqrt{\lambda_{2}} \zeta}-\sqrt{\lambda_{2}{ }^{3}} A_{4} e^{-\sqrt{\lambda_{2}} \zeta}+\sqrt{\lambda_{3}{ }^{3}} A_{5} e^{\sqrt{\lambda_{3}} \zeta}- \\
\sqrt{\lambda_{3}{ }^{3}} A_{6} e^{-\sqrt{\lambda_{3}} \zeta}=0
\end{gathered}
$$

Equations (42) through (47) make a homogenous system of equation which can be expressed in a matrix form as follows:

$$
\left[\begin{array}{cccccc}
1 & 1 & 1 & 1 & 1 & 1 \\
\sqrt{\lambda_{1}} & -\sqrt{\lambda_{1}} & \sqrt{\lambda_{2}} & -\sqrt{\lambda_{2}} & \sqrt{\lambda_{3}} & -\sqrt{\lambda_{3}} \\
\sqrt{\lambda_{1}{ }^{3}} & -\sqrt{\lambda_{1}{ }^{3}} & \sqrt{\lambda_{2}{ }^{3}} & -\sqrt{\lambda_{2}{ }^{3}} & \sqrt{\lambda_{3}{ }^{3}} & -\sqrt{\lambda_{3}{ }^{3}} \\
e^{\sqrt{\lambda_{1}} \zeta} & e^{-\sqrt{\lambda_{1}} \zeta} & e^{\sqrt{\lambda_{2}} \zeta} & e^{-\sqrt{\lambda_{2}} \zeta} & e^{\sqrt{\lambda_{3}} \zeta} & e^{-\sqrt{\lambda_{3}} \zeta} \\
\sqrt{\lambda_{1}} e^{\sqrt{\lambda_{1}} \zeta} & -\sqrt{\lambda_{1}} e^{-\sqrt{\lambda_{1}} \zeta} & \sqrt{\lambda_{2}} e^{\sqrt{\lambda_{2}} \zeta} & -\sqrt{\lambda_{2}} e^{-\sqrt{\lambda_{2}} \zeta} & \sqrt{\lambda_{3}} e^{\sqrt{\lambda_{3}} \zeta} & -\sqrt{\lambda_{3}} e^{-\sqrt{\lambda_{3}} \zeta} \\
\sqrt{\lambda_{1}{ }^{3}} e^{\sqrt{\lambda_{1}} \zeta} & -\sqrt{\lambda_{1}{ }^{3}} e^{-\sqrt{\lambda_{1}} \zeta} \sqrt{\lambda_{2}{ }^{3}} e^{\sqrt{\lambda_{2}} \zeta} & -\sqrt{\lambda_{2}{ }^{3}} e^{-\sqrt{\lambda_{2}} \zeta} \sqrt{\lambda_{3}{ }^{3}} e^{\sqrt{\lambda_{3}} \zeta} & -\sqrt{\lambda_{3}{ }^{3}} e^{-\sqrt{\lambda_{3}} \zeta}
\end{array}\right]\left\{\begin{array}{l}
A_{1} \\
A_{2} \\
A_{3} \\
A_{4} \\
A_{5} \\
A_{6}
\end{array}\right\}=
$$

A non-trivial solution of (48) exists if the determinant of the coefficient matrix is zero. By looking at the coefficient matrix, it is clear that all terms are functions of $\lambda_{r}(r=1,2,3)$. Also, since $\lambda_{r}$ are functions of frequency $\omega$; thus, determinant of the coefficient matrix is also and only a function of $\omega$. By setting the determinant to zero, the natural frequencies of the system will be derived. Associated constants $A_{i}(i=1-6)$ for (48) now can be evaluated corresponding to each natural frequency.

\section{B. Fixed Supports}

In this scenario, at each node, radial and tangential deflections are zero $u=w=0$. The slope and bending moment for the $\mathrm{i}^{\text {th }}$ support must be the same when it is calculated based on either $i^{\text {th }}$ or $(i+1)^{\text {th }}$ segment. Hence, the boundary conditions regarding the slope and bending moment are both continuous along the rim. Mathematically, it is expressed in (49) through (52) $(k=1,2, \ldots, n-1)$.

$$
\begin{aligned}
& u_{i}\left(\theta_{i}\right)=0 \\
& w_{i}\left(\theta_{i}\right)=0
\end{aligned}
$$

$$
\begin{aligned}
& \left.\left.\left(\frac{\partial^{2} w_{k+1}}{\partial \theta^{2}}+w_{k+1}\right)\right]_{\theta=\theta_{i}}=\left(\frac{\partial^{2} w_{k}}{\partial \theta^{2}}+w_{k}\right)\right]_{\theta=\theta_{i}+\zeta} \\
& \left.\left.\left(\frac{\partial^{3} w_{k+1}}{\partial \theta^{3}}+\frac{\partial w_{k+1}}{\partial \theta}\right)\right]_{\theta=\theta_{i}}=\left(\frac{\partial^{3} w_{k}}{\partial \theta^{3}}+\frac{\partial w_{k}}{\partial \theta}\right)\right]_{\theta=\theta_{i}+\zeta}
\end{aligned}
$$

For simplicity of calculation, separate local coordinates is considered for studying each segment. For instance, for the $\mathrm{k}^{\text {th }}$ segment, one can apply the boundary conditions (49) through (52) as follows:

Zero tangential deflection at the beginning of the segment $\theta=0$ :

$$
\sum_{i=1}^{6} A_{k, i}=A_{k, 1}+A_{k, 2}+A_{k, 3}+A_{k, 4}+A_{k, 5}+A_{k, 6}=0
$$

Zero tangential deflection at the beginning of the segment $\theta=\zeta$ :

$$
\begin{gathered}
\sum_{i=1}^{6} A_{k, i} e^{\Upsilon_{i} \zeta}=A_{k, 1} e^{\Upsilon_{1} \zeta}+A_{k, 2} e^{\Upsilon_{2} \zeta}+A_{k, 3} e^{\Upsilon_{3} \zeta}+ \\
A_{k, 4} e^{\Upsilon_{4} \zeta}+A_{k, 5} e^{\Upsilon_{5} \zeta}+A_{k, 6} e^{\Upsilon_{6} \zeta}=0
\end{gathered}
$$
0 :

Zero radial deflection at the beginning of the segment $\theta=$

$$
\begin{aligned}
\sum_{i=1}^{6} \Upsilon_{i} A_{k, i}= & \Upsilon_{1} A_{k, 1}+\Upsilon_{2} A_{k, 2}+\Upsilon_{3} A_{k, 3}+\Upsilon_{4} A_{k, 4}+ \\
& \Upsilon_{5} A_{k, 5}+\Upsilon_{6} A_{k, 6}=0
\end{aligned}
$$

Zero radial deflection at the end of the segment $\theta=\zeta$ :

$$
\begin{gathered}
\sum_{i=1}^{6} \Upsilon_{i} A_{k, i} e^{\Upsilon_{i} \zeta}=\Upsilon_{1} A_{k, 1} e^{\Upsilon_{1} \zeta}+\Upsilon_{2} A_{k, 2} e^{\Upsilon_{2} \zeta}+ \\
\Upsilon_{3} A_{k, 3} e^{\Upsilon_{3} \zeta}+\Upsilon_{4} A_{k, 4} e^{\Upsilon_{4} \zeta}+\Upsilon_{5} A_{k, 5} e^{\Upsilon_{5} \zeta}+\Upsilon_{6} A_{k, 6} e^{\Upsilon_{6} \zeta}=0
\end{gathered}
$$

Continuous slope at the studied node:

$$
\sum_{i=1}^{6}\left(\Upsilon_{i}^{2}+1\right)\left(A_{k, i} e^{\Upsilon_{i} \zeta}-A_{k, i+1}\right)=0
$$

Continuous bending moment at the studied node:

$$
\sum_{i=1}^{6}\left(\Upsilon_{i}^{3}+1\right)\left(A_{k, i} e^{\Upsilon_{i} \zeta}-A_{k, i+1}\right)=0
$$

Equations (53) through (58) can be represented in matrix form by multiplying the parameter matrix to the coefficient one. The order of the parameter matrix is $6 n \times 6 n$ where $n$ is the number of segments.

$[G(\omega)]\left\{A_{11} A_{12} A_{13} A_{14} A_{15} A_{16} \cdots A_{n 1} A_{n 2} A_{n 3} A_{n 4} A_{n 5} A_{n 6}\right\}^{T}=$

A non-trivial solution of (59) exists if the determinant of matrix $G(\omega)$ is zero. By setting the determinant of this matrix to zero, the natural frequencies of the system will be derived. Associated constants $A_{k i}(k=1-n, i=1-6)$ for the mode shape function can be evaluated corresponding to each natural frequency. For instance, for the natural frequency $\omega_{\mathrm{r}}$, there will be $6 n$ mode shape constants $A_{k i}$ which $6 n-1$ of them can be expressed based on the remaining one.

\section{RESULTS AND CONCLUSION}

The dynamic behavior of a symmetrically supported thin ring was studied in the current work. This analysis was performed for a ring with two types of boundary conditions i.e. hinged and fixed supports. Having used the Abaqus package, several numerical examples were worked out to show the accuracy of the analytical solutions. The evaluated first three natural frequencies for those cases are shown in Table 1. The reason leading to the slight differences between the analytical 
and the FEM results might be due to the simplification applied in the mathematical model such as neglecting the effects of the rotary inertia and shear deformations.

Table 1. Comparison of natural frequencies obtained from the analytical method and FEM.

\begin{tabular}{cc|cccccc}
\hline \multicolumn{2}{c|}{$\begin{array}{c}\text { Type and } \\
\text { Number of }\end{array}$} & \multicolumn{2}{c}{$\omega_{1}$ (Hertz) } & \multicolumn{2}{c}{$\omega_{2}$ (Hertz) } & \multicolumn{2}{c}{$\omega_{3}$ (Hertz) } \\
\cline { 2 - 8 } \multicolumn{2}{c}{$\begin{array}{c}\text { Supports } \\
\text { Hinged }\end{array}$} & Exact & FEM & Exact & FEM & Exact & FEM \\
\hline \multirow{2}{*}{ Hing } & 12.20 & 12.27 & 36.20 & 36.79 & 55.87 & 57.10 \\
& 4 & 23.99 & 24.10 & 42.98 & 44.04 & 84.02 & 86.78 \\
& 5 & 38.87 & 39.14 & 54.01 & 55.51 & 113.25 & 119.86 \\
\hline \multirow{2}{*}{ Fixed } & 3 & 30.10 & 30.32 & 48.12 & 49.45 & 86.98 & 89.04 \\
& 4 & 41.83 & 42.10 & 54.21 & 55.43 & 102.82 & 104.56 \\
& 5 & 55.02 & 55.52 & 59.98 & 60.21 & 117.54 & 120.10 \\
\hline
\end{tabular}

Fig. 2 shows the deflected ring for some picked scenarios from the above table. Comparing the cases of hinged and fixed supports, both analytical and FEM methods confirmed that the natural frequencies for the case of having fixed supports are considerably higher. This behavior could be also predicted based on the physical model of the problem.

a.

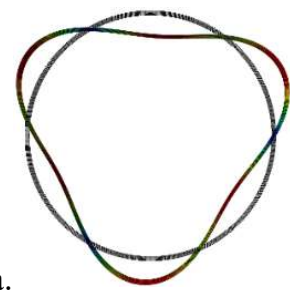

c.
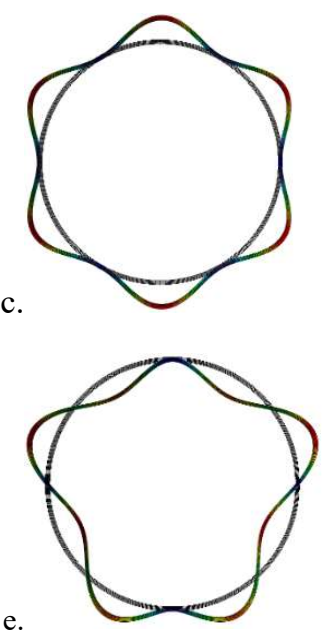

e.

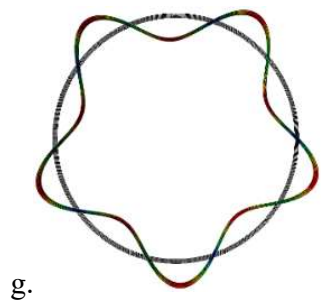

b.

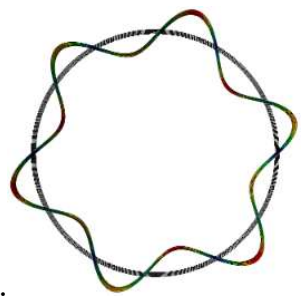

d.

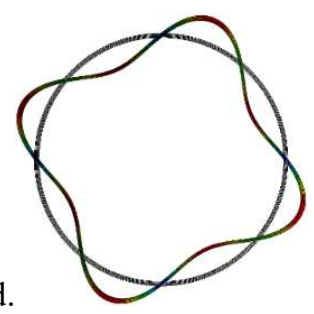

f.

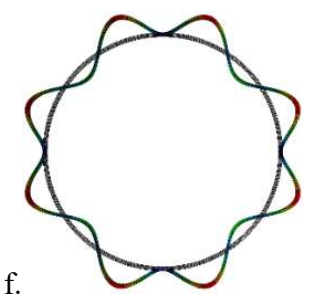

h.

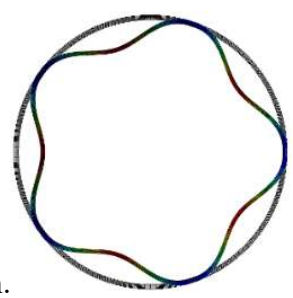

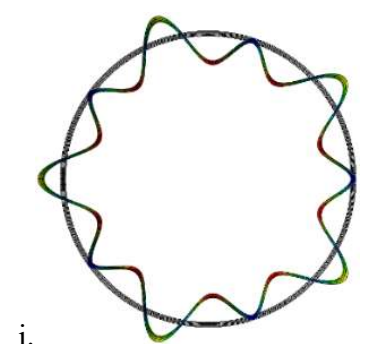

Figure 2. Mode shapes for a thin ring having 3 (a-c), 4 (d-f), and 5 (g-i) hinged supports.

For lower frequencies, the precision of the natural frequencies derived from the formulation in the current work is pretty close to the FEM results. In the formulation of the governing equations of this work, a number of simplifying assumptions have made. These assumptions result in observing some differences in higher natural frequencies. Results obtained from this analysis are in excellent agreement with those found from FEM analysis in the Abaqus software.

\section{RECOMMENDATION FOR FUTURE WORK}

For future work, it is recommended to study the behavior of Timoshenko rings under different boundary conditions. Moreover, the bolted supports for either Euler-Bernoulli or Timoshenko beams can be studied and then the obtained results can be compared with results expressed in the current work.

\section{REFERENCES}

[1] R. Hoppe, "The bending vibration of circular ring", Crelle Journal of Mathematics 73, 158, 1871.

[2] S.S. Rao, "Vibration of continuous systems", Wiley Publication, ISBN: 0471771716, 2007.

[3] X.H. Wu, R.G. Parker, "Vibrations of rings on a general elastic foundation", Journal of Sound and Vibration 295, 2006.

[4] D.S. Zhang, S.Y. Wang, J.P. Liu, "Analytical prediction for free response of rotationally ring-shaped periodic structures", ASME Journal of Vibration and Acoustics 136, 2014.

[5] T.E. Lang, "Vibration of thin circular rings", Jet Propulsion Laboratory Technical Report No. 32-261, 1962.

[6] R. E. Ball, “. Dynamic analysis of rings by finite-differences", Journal of Engineering Mechanics Division, American Society of Civil Engineers 93, 1-10,1967.

[7] K. Singh, B.L. Dhoopar, Free vibration of circular rings on radial supports, Journal of Sound and Vibration 65 (1979) 297-301.

[8] F.M. Detinko, "Free vibration of a thick ring on multiple supports", International Journal of Engineering Science 27 (1989) 1429-1438.

[9] D. Allaei, W. Soedel, T.Y. Yang, "Natural frequencies and modes of rings that deviate from perfect axisymmetry", Journal of Sound and Vibration 111 (1986) 9-27.

[10] S.S. Rao, V. Sundararajan, "In-plane flexural vibration of circular rings", Journal of Applied Mechanics, Design Data and Methods 36 (1969) 620625.

[11] K.B. Sahay, V. Sundararajan, "Vibration of a stiffened ring considered as a cyclic structure", Journal of Sound and Vibration 22 (1972) 467-473.

[12] T.J. McDaniel, "Dynamics of Circular Periodic Structures", Journal of Aircraft 8 (1971) 143-149. 
[13] V.R. Murthy, N.C. Nigam, "Dynamic characteristics of stiffened rings by transfer matrix approach", Journal of Sound and Vibration 39 (1975) 237-245.

[14] K. Singh, B.L. Dhoopar, "Free vibration of circular rings on radial supports", Journal of Sound and Vibration 65 (1979) 297-301.

[15] A.K. Mallik, D.J. Mead, "Free vibration of thin circular rings on periodic radial supports", Journal of Sound and Vibration 54 (1977) 13-27.

[16] A.A. Zakrzhevskii, V.F. Tkachenko, V.S. Khoroshilov, "Natural modes and frequencies of in-plane vibrations of a fixed elastic ring", International Applied Mechanics, Vol. 46 No. 12, May 2011.

[17] S. Lipschutz, M.R. Spiegel, J. Liu, "Schaum's Outline of Mathematical Handbook of Formulas and Tables", $5^{\text {th }}$ edition, McGraw Hill Publication, ISBN: 978-1260010534, Oct 2017. 